\title{
Histometric data obtained by in vivo confocal laser scanning microscopy in patients with systemic sclerosis Kirsten Sauermann ${ }^{* 1,2}$, Thilo Gambichler ${ }^{1}$, Sören Jaspers ${ }^{2}$, Michael Radenhausen ${ }^{1}$, Solveig Rapp ${ }^{1}$, Susanne Reich ${ }^{1}$, Peter Altmeyer ${ }^{1}$, Sven Clemann ${ }^{1}$, Stefan Teichmann ${ }^{2}$, Joachim Ennen ${ }^{2}$ and Klaus Hoffmann ${ }^{1}$
}

Address: ${ }^{1}$ Department of Dermatology, Ruhr-University Bochum, Gudrunstrasse 56, D-44791 Bochum, Germany and ${ }^{2}$ Research \& Development Cosmed, Beiersdorf AG, KST 4223, Unnastrasse 48, Hamburg, Germany

E-mail: Kirsten Sauermann* - kirsten.sauermann@beiersdorf.com; Thilo Gambichler - t.gambichler@derma.de;

Söeren Jaspers - soeren.jaspers@beiersdorf.com; Michael Radenhausen - m.radenhausen@ derma.de; Solveig Rapp - s.rapp@derma.de;

Susanne Reich - s.reich@derma.de; Peter Altmeyer - p.altmeyer@derma.de; Sven Clemann - sven.clemann@beiersdorf.com;

Stefan Teichmann - stefan.teichmann@beiersdorf.com; Joachim Ennen - joachim.ennen@beiersdorf.com;

Klaus Hoffmann - k.hoffmann@derma.de

${ }^{*}$ Corresponding author

Published: 6 August 2002

BMC Dermatology 2002, 2:8
Received: I November 200 I

Accepted: 6 August 2002

This article is available from: http://www.biomedcentral.com/I47I-5945/2/8

(C) 2002 Sauermann et al; licensee BioMed Central Ltd. This article is published in Open Access: verbatim copying and redistribution of this article are permitted in all media for any non-commercial purpose, provided this notice is preserved along with the article's original URL.

\begin{abstract}
Background: It would be a benefit if time-saving, non-invasive methods could give hints for diagnosing systemic sclerosis. To investigate the skin of patients with systemic sclerosis using confocal laser scanning microscopy in vivo and to develop histometric parameters to describe characteristic cutaneous changes of systemic sclerosis observed by this new technique, we conducted an exploratory study.
\end{abstract}

Materials and Methods: Fifteen patients with systemic sclerosis treated with extracorporal photopheresis were compared with 15 healthy volunteers and 10 patients with other disorders also treated with extracorporal photopheresis. All subjects were investigated using confocal laser scanning microscopy in vivo.

Results: Micromorphologic characteristics of skin of patients with systemic sclerosis and measuring parameters for melanisation, epidermal hypotrophy, and fibrosis for dislocation of capillaries by collagen deposits in the papillary dermis were evaluated. An interesting finding was an increased thickness of the tissue in the dermal papillae superior to the first dermal papilla vessel. It was also possible to reproduce characteristic histologic features by confocal laser scanning microscopy in vivo. Histometric parameters for fibrosis and vascular features developed in this study showed significant differences in patients with systemic sclerosis compared to controls.

Conclusions: Although the predominant histopathological features in systemic sclerosis are findings of the reticular dermis and the subcutis, and in histopathological investigation the epidermis seems to remain unaffected by the disease, we have demonstrate some characteristic differences in the epidermis and papillary dermis by confocal laser scanning microscopy in vivo. Some of them have not been described so far. However, to use this technique as a tool for diagnosis and/or staging of systemic sclerosis, further studies are needed investigating the sensitivity and specificity of the histometric parameters developed in this study. 


\section{Background}

Systemic sclerosis (SSc) is a rare chronic disease of unknown origin, associated with a high morbidity and mortality. It is characterised by excessive deposition of collagen in all involved organs. This leads to sclerosis of the skin and fibrosis in internal organs (e.g., gastrointestinal tract, lungs, kidneys), which often determine the prognosis of the disease. The pathology of connective tissues is similar in localized scleroderma and SSc $[1,2]$. Two stages have been described in the development of sclerodermatous skin: an early cellular stage and a fibrotic stage [3]. Diagnosis of SSc is based mainly on clinical features and the pattern of antibodies and is difficult in early stages. Therefore it would be a benefit, if time-saving, non-invasive methods could give hints for diagnosing SSc.

In vivo confocal laser scanning microscopy (CLSM) is a new non-invasive microscopy technique with high resolution at a specific depth allowing to image skin on a cellular level in real-time with a penetration depth of about $200 \mu \mathrm{m}$ to $250 \mu \mathrm{m}$ [4]. Contrast is based on reflection at interfaces between two media of different refraction indices. Confocal images are orientated parallel to the skin surface (en face). It has been recently shown that CLSM is a feasible tool for microscopic analysis of skin morphometry in vivo [5-9]. It should be noted in this context, that CLSM allows to study tissues without artefacts by dehydration, fixation and staining of the tissue and that it of- fers the unique possibility to study the vasculature of the skin with blood flow in ist three-dimensional pattern. Micromorphologic changes in live human skin of patients with SSc viewed by CLSM have not been reported so far. This exploratory study was conducted to investigate micromorphologic characteristics of skin in patients with SSc and in healthy persons using CLSM and to develop histometric parameters to describe the differences in vivo. To be sure that the differences between the diseased and the healthy population are not caused by extracorporeal photophoresis (EcP), the therapy the SSc patients were treated with, a second control group, composed of patients receiving the same therapy for another disease, was investigated.

\section{Methods \\ Subjects}

Fifteen patients (13 females/ two males; median age: 54; range: 26-70) with SSc were included into the study after signed informed consent. Inclusionary criterion consisted of cutaneous involvement of the forearms. Since corticosteroids, which are often used in therapy of SSc, show dramatic effects on histometric parameters of the skin, we investigated a group of patients with SSc that were mainly treated with extracorporeal photophoresis (EcP). The patients fulfilled the preliminary criteria for SSc proposed by the American College of Rheumatology [10]; they were

Table I: Characteristics of 15 patients with SSc and data of CLSM in vivo

\begin{tabular}{|c|c|c|c|c|c|c|c|c|c|c|}
\hline No. & Sex & $\begin{array}{c}\text { Age } \\
\text { (years) }\end{array}$ & $\begin{array}{l}\text { Duration } \\
\text { of disease } \\
\text { (years) }\end{array}$ & SSc type 9 & $\begin{array}{l}\text { Reduction of } \\
\text { hand mobility }\end{array}$ & $\begin{array}{l}\text { Pulmonary } \\
\text { involvement }\end{array}$ & $\begin{array}{c}\text { Renal } \\
\text { involvement }\end{array}$ & $\begin{array}{l}\text { Esophagus } \\
\text { involvement }\end{array}$ & EcP (sessions) & PC \\
\hline
\end{tabular}

\begin{tabular}{|c|c|c|c|c|c|c|c|c|c|c|}
\hline 1 & $f$ & 45 & 9 & diffuse & $(+)$ & - & - & + & 80 & $x$ \\
\hline 2 & $f$ & 31 & 2 & diffuse ${ }^{*}$ & + & ++ & - & - & 19 & $x$ \\
\hline 3 & $\mathrm{~m}$ & 36 & 3 & diffuse & + & + & - & + & 13 & 18,2 \\
\hline 4 & $f$ & 47 & I & diffuse & - & + & - & $(+)$ & 7 & 15,7 \\
\hline 5 & $f$ & 54 & II & diffuse & - & + & + & ++ & ?? & 22,2 \\
\hline 6 & $f$ & 60 & 4 & diffuse & - & ++ & + & + & 15 & 14,0 \\
\hline 7 & $\mathrm{~m}$ & 56 & 4 & diffuse & + & - & - & $(+)$ & 34 & 15,0 \\
\hline 8 & $f$ & 56 & 3 & diffuse & $(+)$ & - & + & ++ & 10 & 18,0 \\
\hline 9 & $f$ & 32 & I & limited & - & - & - & - & 3 & 18,3 \\
\hline 10 & $f$ & 68 & I & limited & $(+)$ & - & - & - & I & 18,2 \\
\hline II & $f$ & 60 & 2 & limited & - & - & - & - & 8 & $x$ \\
\hline 12 & $f$ & 70 & I & diffuse & $(+)$ & ++ & - & + & 4 & 22,7 \\
\hline 13 & $f$ & 26 & 9 & diffuse & + & ++ & - & ++ & 3 & 47,4 \\
\hline 14 & $f$ & 61 & 5 & diffuse & + & ++ & - & ++ & 35 & 24,4 \\
\hline 15 & $f$ & 54 & 21 & diffuse & + & + & - & ++ & 1 & 28,8 \\
\hline
\end{tabular}

${ }^{*}$ CREST syndrome; \# EcP combined with azathioprine and prednisolone; scoring: none $=-$, mild $=(+)$, moderate $=+$, severe $=++; x=$ not investigated 
grouped according to the classification system by LeRoy et al. [11]; three patients had limited type, and twelve patients had diffuse type of SSc. The median duration of disease was three years (range: 1-21). The patients' profiles are summarized in detail in Table 1. In all patients, EcP was performed with UVAR Photopheresis Equipment (Therakos LtD, West Chester, Pa), as described before in detail [12]. EcP sessions were performed on two consecutive days a months. In three cases (patient no.: 2, 7, 14) EcP was combined with $200 \mathrm{mg}$ azathioprine plus $5 \mathrm{mg}$ prednisolone per day.

In addition, 15 healthy volunteers (14 female, one male), median age 50 (range: 23-75) served as a control group. Ten patients getting EcP treatment because of other diseases (six patients with atopic dermatitis, one patient with graft versus host reaction, two patients with T-cell lymphoma, one patient with dermatomyositis, median age 36 , range $22-72$ ) served as a second control group for some of the parameters measured. Written informed consent was obtained from all subjects prior to participation in this study.

\section{In vivo confocal laser scanning microscopy (CLSM)}

A commercially available CLSM (Vivascope 1000; Lucid Inc, Henrietta, NY; [http://www.lucid-tech.com] was used for in vivo confocal laser scanning microscopy. This microscope uses a $830 \mathrm{~nm}$ gallium-arsenid laser operating at a power less than $30 \mathrm{~mW}$ at the tissue surface and a water immersion objective. Detailed description of the confocal microscope has also been reported previously $[4,13]$. The Vivascope 1000 allows to image skin to a depth of $200 \mu \mathrm{m}$ in vivo, non-invasively with a lateral resolution of $0.4 \mu \mathrm{m}$ and $1.9 \mu \mathrm{m}$ horizontal. With 20 images per second it is possible to study the epidermis and papillary dermis on a cellular level in real-time-imaging. The field of view shows $128 \mu \mathrm{m} \times 260 \mu \mathrm{m}$. In all subjects all measurements were performed on a skin site of $1.5 \mathrm{~mm} \times 1.5 \mathrm{mmright}$ in the middle of the right ventral forearm.

Additionally to the investigation of the morphology of the tissue the following parameters were assessed histomorphometrically: The thickness of stratum corneum (DSC), measured from skin surface to the first recognizable nucleus in the granular layer [14]. The minimal thickness of epidermis $\left(\mathrm{E}_{\mathrm{min}}\right)$, measured as the distance between skin surface and the most apical recognizable dermal structure; four measurements per patient were performed for DSC and $\mathrm{E}_{\min }[14]$. The contrast between stratum basale and stratum spinosum - a semiquantitative parameter for melanin content (MI) [15]. At least 10 calculations per patient were performed from images captured during the investigation. The dermal reflectivity index (DI), the contrast between stratum spinosum and papillary dermis is a parameter for the reflectivity of dermis.
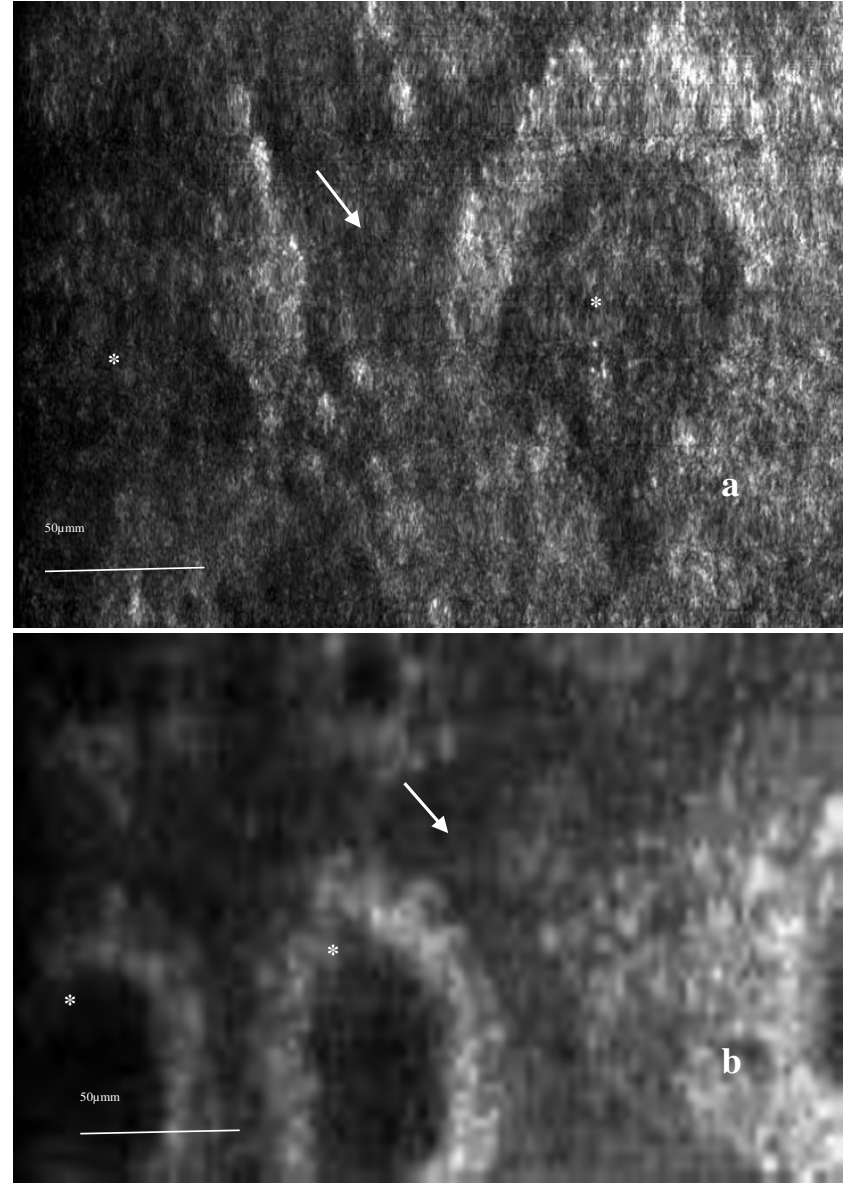

Figure I

Virtual section through the skin of a SSc patient (a) and of a healthy volunteer (b) at the height of the epidermal-dermal junction. The basal layer appears as bright circles (due to the strong reflection of melanosomes) around dermal papillae (*). These circles are surrounded by spinous layer (arrowhead), which exhibits a brighter aspect than the dermal tissue normally, but not so in the SSc patient due to fibrosis.

Ten measurements per patient were performed. The intensities of MI and DI were assessed from captured images of the skin with the focus plane at the height of the epidermal-dermal junction by the aid of the image analysis software (IMAGE TOOL, UTHSCA, San Antonio). These relative parameters were developed because the absolute intensity of the structures depends on the other factors such as the reflectiveness and thickness of the horny layer, where light is absorbed and scattered in a varying amount and the signals from deeper structures are influenced. By comparing the intensity of the basal layer or papillary dermis with the quite stable signal of the surrounding spinous layer, changes in the amount of melanosomes and collagen, the main substances or particles in the basal layer and the dermis providing reflection can be estimated $[14,16]$. 
The distance between the top of the dermal papillae and the capillary with visible blood flow (PC) was measured by means of the micronscrew in five papillae per patient, but only in twelve out of fifteen patients with SSc investigated as the parameters PC and DI were developed during this study.

\section{Statistics}

Results are expressed as means \pm SD. Statistical significance of the data was calculated by using the two-tailed Student's t test to determine the difference between the mean values of the micromorphometric measurements obtained in patients with SSc and healthy subjects. P values of less than 0.05 were considered significant. As PC was not normally distributed in the SSc group of patients, a Mann-Whitney rank sum test was used to calculate the statistical significance of differences between the control groups and the SSc group.

\section{Results}

In the skin of the SSc patients, the epidermis showed a normal differentiation pattern. DSC showed no significant $(\mathrm{p}=0.36)$ difference between SSc $(11.0 \pm 2.6 \mu \mathrm{m})$ and healthy skin $(10.4 \pm 2.0 \mu \mathrm{m})$. In comparison to the healthy controls $(39.4 \pm 5.5 \mu \mathrm{m})$ and the EcP control group $(37.1 \pm 6.8 \mu \mathrm{m})$ there was a significant increase $(\mathrm{p}$ $=0.049$ and $\mathrm{p}=0.025)$ for $\mathrm{E}_{\min }$ in SSc patients $(44.0 \pm 7.0$ $\mu \mathrm{m})$. There was no significant difference $(\mathrm{p}=0.38)$ between the healthy control group and the EcP control group. In the skin of the SSc patients, the basal layer surrounding the dermal papillae, which were slightly increased in number, showed a bright reflection and a strong contrast compared with the surrounding spinous layer. The MI was significantly higher $(\mathrm{p}=0.0008)$ in the group of SSc patients than in the control group (median $1.79 \pm 0.24$ compared to $1.33 \pm 0.16$ ). No significant differences could be found between the EcP control group $(1.54 \pm 0.32)$ and either the SSc group $(\mathrm{p}=0.07)$ or the healthy control $(\mathrm{p}=0.32)$.

In the papillary dermis of the healthy control group a network of collagen fibers could be observed. Bundles of fibers showed a brighter reflection than the surrounding matrix. Compared to the images of the control groups, the papillary dermis in the skin of the SSc patients showed a higher reflectivity and appeared more homogenous (Fig. 1 ), only few discrete collagen fiber bundles could be distinguished from the surrounding. The dermal papillae exhibited nearly the same level of reflectiveness as the surrounding spinous layer. In contrast, in healthy skin, they were less reflective than the tissue in the spinous layer. DI was significantly increased in the disease group compared to both of the control groups $[1.06 \pm 0.20$ in the SSc patients compared to $0.59 \pm 0.10$ in the healthy control group $\left(\mathrm{p}<10^{-6}\right)$ and $0.52 \pm 0.15$ in the $\mathrm{EcP}$ group

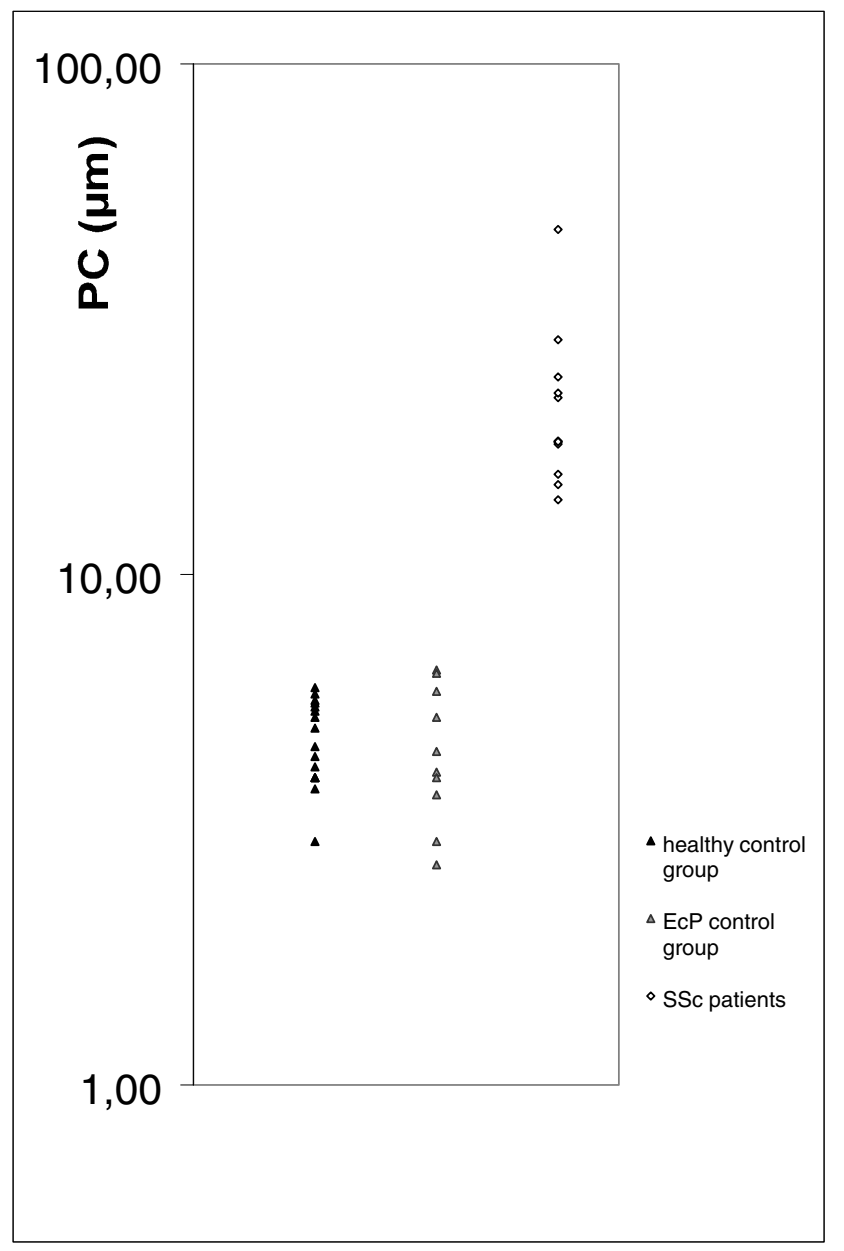

Figure 2

The distance between the top of the dermal papillae and a capillary showing blood flow (PC) is increased in the skin of SSc patients. None of the SSc patients showed PC distances in the range of the control groups.

$\left.\left(\mathrm{p}<10^{-6}\right)\right]$. No significant difference was found between the two control groups $(\mathrm{p}=0.27)$.

The distance between the top of the dermal papillae and the capillary loop with blood flow was significantly increased to $18.1 \mu \mathrm{m}$ in median with an higher quartile at $22.7 \mu \mathrm{m}$ and a lower quartile at $18.0 \mu \mathrm{m}$ in the SSc patients compared to $4.8 \mu \mathrm{m} \pm 0,9 \mu \mathrm{m}$ in the healthy control group $(\mathrm{p}<0.001)$ and $4.6 \pm 1.3 \mu \mathrm{m}$ in the EcP control group ( $<<0.001$ ) (Fig. 2). No significant difference could be found between both control groups $(\mathrm{p}=0.74)$. Vessels at the height of the upper dermal plexus were often narrowed in their lumina and showed a decreased speed of blood flow. The surrounding tissue showed a strong reflection. Occasionally, tortuous, multiple cappillaries with a slightly increased diameter could be observed in a 

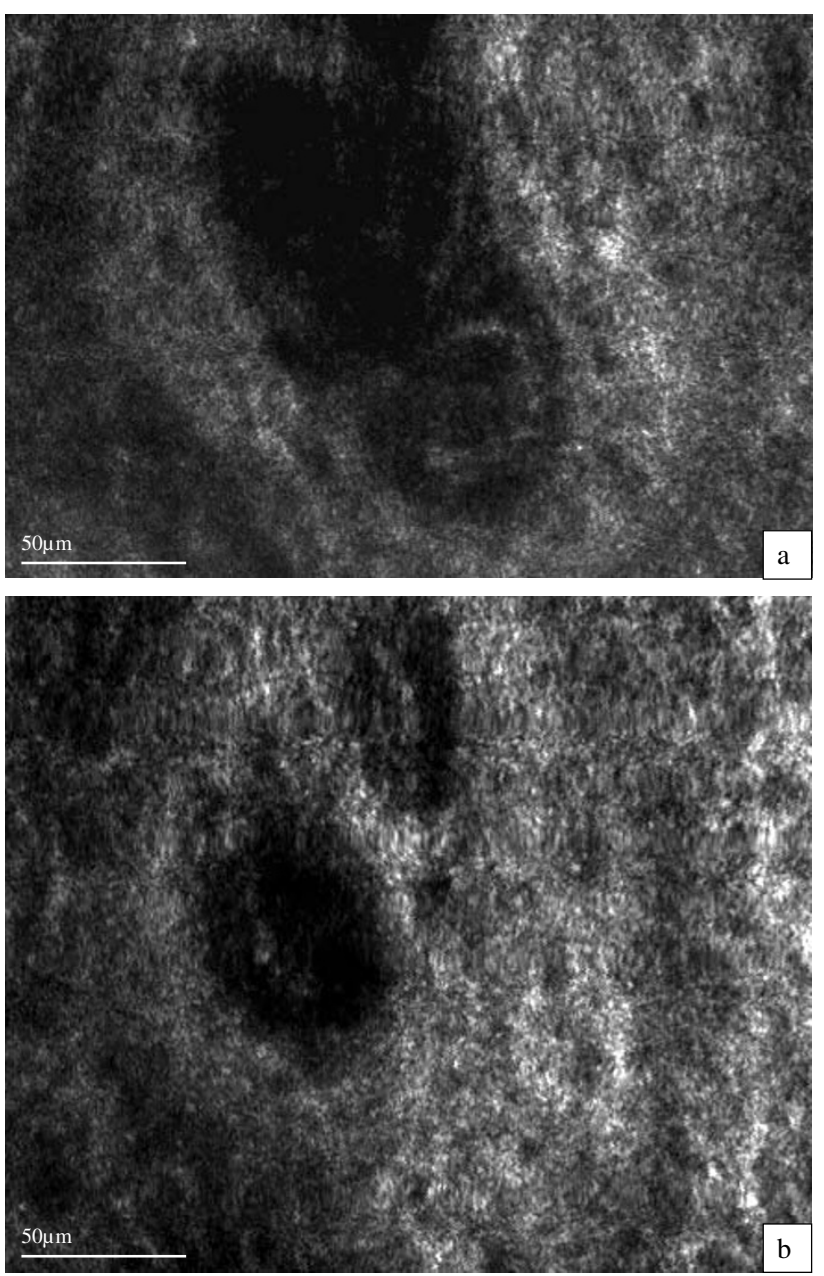

Figure 3

A dermal papilla in the skin of a SSc patient in $55 \mu \mathrm{m}$ (a) and $65 \mu \mathrm{m}$ (b) depth. Tortuous, multiple cappillaries showing dark vessel walls and bright blood cells in the lumina (arrows) could be observed in a dermal papillae in the skin of SSc patients, whereas in the control groups, only one capillary loop per papillae could be found.

dermal papillae (Fig. 3), whereas in the control groups, only one capillary loop per papillae could be observed.

\section{Discussion}

Although the epidermis of patients with SSc is generally rather unaffected in histopathological investigation [17], in late stages a dissappearance of the rete ridges can be observed occasionally [17]. This might be the explanation for the increase in the minimal epidermal thickness in SSc patients compared to the healthy control group, as this parameter is influenced by the height of the epidermaldermal junction. It increases, for example, with age, too, when the epidermal-dermal junction flattens (as we have described before) and therefore the significant difference between the minimal epidermal thickness in the SSc group and the younger EcP control group could be at least in part due to the difference in age [14]. The differences between the healthy control group and the SSc patients can not be explained by this effect.

The increased reflectivity of the basal layer measured by the MI in the SSc group could reflect the hyperpigmentation due to the chronic inflammation of the skin of these patients. Although postinflammatory hyperpigmentation is characterized in histological sections by loss of melanin into the dermis, which would probably not influence this parameter, there seems to be an activation of melanocytes leading to a higher amount of melanosomes in the basal layer, too. As this occured in the skin of patients of the EcP control group with atopic dermatitis, too, it does not surprise, that the MI in this control group showed a large variation and no level of significance for the difference from the two other groups investigated could be achieved $(\mathrm{p}=$ 0.07 ). Anyway, there is no proof that the hyperpigmentation is not at least in part due to the increased light sensitivity induced by psoralens, taken up during EcP.

In the papillary dermis of healthy volunteers, collagen fibers appear as a loosely arranged, medium bright network in confocal images. The more homogenous appearance and brighter reflection in the papillary dermis of the SSc patients may be due to a different arrangement and an increase of collagen fibers. The fibrosis of the papillary dermis can be measured semiquantitatively by the relative reflectiveness of the dermis (DI). It is unlikely that these features are due to therapy by EcP as the control group of patients treated with EcP did not show similar features either.

Restriction of the blood flow in the lumina of the vessels in the reticular dermis by fibrotic walls and a reduction of the number of vessels in the fibrotic collagen are common histopathological features in SSc [17]. The increase of PC reflects this restriction of blood vessels by fibrosis. There seems to be a cap of collagen built up in the tops of the dermal papillae, seperating the capillaries from the epidermis. The decreased perfusion of the tissue may lead to hypoxia and frustrane angiogenesis, which could result in the multiple capillaries coils observed occasionally [18]. As all SSc patients investigated showed dramatic increase in PC, the distance between the top of the dermal papillae and a capillary with blood flow, PC seems to be a sensitive parameter for diagnosing SSc. No member of the control groups exhibited data in a similar, high range of this parameter. However, there is a need for further prospective studies to investigate its promising specificity. As the patients (number 13, 14 and 15) with severe involvement of the pulmonary function and the esophagus and patient nunber 5 with mild disturbance of the pulmonary func- 
tion and severe involvement of the esophagus all got the feature of a relatively high PC, this parameter could possibly serve as a non-invasive method in staging of the fibrosis and controlling disease advance and effectiveness of therapy, too.

\section{Conclusions}

Although the predominant histopathological findings of SSc are features of the reticular dermis and the subcutis, and in histopathological investigation the epidermis seems to stay unaffected by the disease [17], we have shown some characteristic differences in the epidermis and papillary dermis by CLSM in vivo. Further studies are needed to investigate the sensitivity and specificity of the differences observed, especially of PC and DI, in order to use in vivo CLSM as a tool for diagnosis and/or staging of SSc. Although this study can only be the first step in the long process of diagnostic evaluation of the morphological characteristics, we would like to postulate that CLSM is well suitable for histometric studies on skin - it permits repetitive investigations, and it is a promising technique for dermatological research.

\section{List of abbreviations}

Systemic sclerosis: SSc; confocal laser scanning microscopy: CLSM; extracorporeal photophoresis: EcP; thickness of stratum corneum: DSC; minimal thickness of epidermis: $\mathrm{E}_{\min }$; melanin content: MI; dermal reflectivity index: DI; distance between the top of dermal papillae and capillary with visible blood flow: PC

\section{Competing interests}

None declared.

\section{References}

I. Altman RD, Medsger TA, Bloch DA, Michel BA: Predictors of survival in systemic sclerosis (scleroderma). Arthritis Rheum I991, 34:403-413

2. Sollberg S, Krieg T: Systemische Sklerodermie. Hautarzt 1995, 46:587-60।

3. Torres JE, Sanchez JL: Histopathologic differentiation between localized and systemic scleroderma. Am J Dermatopathol 1998, 20:242-245

4. Imbert D, Hoogstraate J, Marttin E, Cullander C: Imaging thick tissues with confocal microscopy. Methods Mol Biol 1999, I 22:34I355

5. Huzaira M, Rius F, Rajadhaksha M, Anderson RR, González S: Topographic variations in normal skin, as viewed by in vivo reflectance confocal microscopy. Invest Dermatol 200 I, I I 6:846-852

6. González S, González E, White WM, Rajadhyksha M, Anderson R: Allergic contact dermatitis: correlation of in vivo confocal imaging to routine histology. I Am Acad Dermatol 1999, 40:708-7I3

7. Sauermann K, Clemann S, Jaspers S, Maerker U, Gambichler T, Altmeyer P, Ennen J, Hoffmann K: Changes of human skin caused by occlusion and low humidity investigated with histometric measurements by confocal laser scanning microscopy in vivo. Skin Res Technol, in press

8. González S, Rajadhyaksha M, Rubinstein G, Anderson RR: Characterization of psoriasis in vivo by reflectance confocal microscopy. I Med 1999, 30:337-356

9. Sauermann K, Jaspers S, Clemann S, Hoffmann K, Ennen J: Confocal laser scanning microscopy in vivo: a promising tool in skin research. Cell Mol Biol 2000, 46:late abstracts
10. Subcommitee for Scleroderma Criteria of the American Rheumatism Association Diagnostic and Therapeutic Criteria Committee: Preliminary criteria for the classification of systemic sclerosis (scleroderma). Arthritis Rheum 1980, 23:581-590

II. LeRoy EC, Krieg T, Black C, Medsger TA jr, Fleischmajer R, Rowell $\mathrm{N}$, et al: Scleroderma (systemic sclerosis): classification, subset, and pathogenesis. J Rheumatol 1988, I 5:202-205

12. Edelson RL, Berger C, Gasparro FP, Jegasothy BV, Heald P, Wintroub $B$, et al: Treatment of cutaneous T-cell lymphoma by extracorporeal photochemotherapy. N Engl J Med 1987, 3 16:297-303

13. Rajadhyaksha M, Gonzaléz S, Zavislan J, Anderson RR, Webb RH: In vivo confocal scanning laser microscopy of human skin II. Advances in instrumention and comparison to histology. J Invest Dermatol 1999, I 1 3:293-303

14. Sauermann K, Clemann S, Jaspers S, Gambichler T, Altmeyer P, Hoffmann K, Ennen J: Age related changes of human skin investigated with histometric measurements by confocal laser scanning microscopy in vivo. Skin Res Technol 2002, 8:52-56

15. Sauermann K, Jaspers S, Ennen J, Clemann S, Uhlmann B, Gers-Barlag $\mathrm{H}$, Hoffmann K: Histometric data obtained by in vivo confocal laser scanning microscopy Skin Res Technol 2000, 6: I47

16. Rajadhyaksha M, Grossman M, Esterowitz D: In vivo confocal scanning laser microscopy of human skin: melanin provides strong contrast. J Invest Dermatol 1995, 104:946-52

17. David Elder: Lever's Histopathology of the skin 8th edition, Philadelphia, New York, Lippincott-Raven Publishers, 1997, p274-278

18. Stern-Kiefer M, Porwol T, Acker H, Altmeyer P, Bacharach-Buhles M: Three dimensional reconstruction in dermatology. A view of new possibilities in the routine histological diagnosis. Hautarzt 2000, 5 I:746-752

\section{Pre-publication history}

The pre-publication history for this paper can be accessed here:

http://www.biomedcentral.com/1471-5945/2/8/prepub

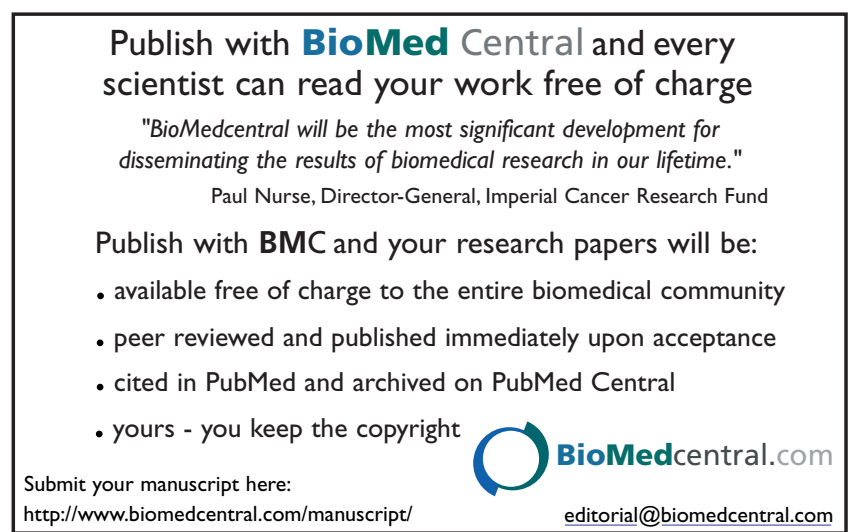

